TRANSACTIONS OF THE

AMERICAN MATHEMATICAL SOCIETY

Volume 195, 1974

\title{
SOME MAPPING THEOREMS
}

\author{
BY \\ R. C. LACHER ${ }^{1}$ )
}

ABSTRACT. Various mapping theorems are proved, culminating in the following result for mappings $f$ from a closed $(2 k+1)$-manifold $M$ to another, $N$ : If "almost all" point-inverses of $f$ are strongly acyclic in dimensions less than $k$ and if "almost all" point-inverses of $f$ have Euler characteristic equal to one, then all but finitely many point-inverses are totally acyclic. (Here "almost all" means "except on a zero-dimensional set in $N "$ ") More can be said when $k=1$ : If $f$ is a monotone map between closed 3-manifolds and if the Euler characteristic of almost-all point-inverses is one, then all but finitely many point-inverses of $f$ are cellular in $M$; consequently $M$ is the connected sum of $N$ and some other closed 3-manifold and $f$ is homotopic to a spine map. Other results include an acyclicity criterion using the idea of "nonalternating" mapping and the following result for PL maps $\phi$ between finite polyhedra $X$ and $Y$ : If the Euler characteristic of each point-inverse of $\phi$ is the integer $c$ then $X(X)=c X(Y)$.

We begin with a clarification of the term "mapping theorem": this is to mean a theorem in which "local" assumptions are made on a map $f$ (i.e., assumptions are made on point-inverses of $f$ ) and "global" conclusions are drawn. The global conclusions may be topological (e.g., deducing that the domain and range of $f$ are homeomorphic) or algebraic (e.g., concluding $f$ has degree \pm 1 ). The classical Vietoris mapping theorem [3] is the best known example of the sort of result we have in mind.

Global conclusions can sometimes be obtained using "finiteness" theorems. As an illustration, consider a map $f: M^{n} \rightarrow \rightarrow N^{n}$ between closed topological manifolds. If the set $C$, of points $y \in N$ for which $f^{-1}(y)$ is not cellular in $M$ can be shown to be finite, $n \neq 4$, then results of S. Armentrout [2] and L. C. Siebenmann [10] imply that $M$ is homeomorphic to the connected sum of $N$ and another manifold. (See [8] for a more complete discussion of finiteness theorems.)

For mappings between closed even-dimensional manifolds there is a finite-

Presented to the Society, January 26, 1973 under the title Maps with locally trivial euler characteristic on prime 3-manifolds are cellular; received by the editors November 16, 1972 and, in revised form, July 24, 1973. 57 L05.

AMS (MOS) subject classifications (1970). Primary 57A15, 57A60; Secondary 57B05,

Key words and phrases. Mapping, acyclic, finiteness, cellularity.

(1) Alfred P. Sloan Fellow, partially supported by grant NSF GP1 9964.

Copyright $\odot$ 1974, American Mathematical Society 
ness theorem as follows (cf. [7]): If each $f^{-1}(y)$ has $(k-1)$-connected shape, $n=2 k$, then $C$, is finite $(k \neq 2)$. In $\$ 2$, after reviewing the even-dimensional case in more detail in $\$ 1$, we give an analogous result for odd dimensions: If each $f^{-1}(y)$ has $(k-1)$-connected shape and Euler characteristic one, $n=2 k+1$, then $C_{f}$ is finite (no restriction on $k$ ). For $n=3$, this generalizes (and uses) a result of A. Wright [17] and thus seems to explain Wright's theorem as a phenomenon about odd-dimensional manifolds.

For functions of a real variable, the concepts of monotone map and nonalternating map coincide. In $\$ 3$, we show how this equivalence is a special case of a general fact for mappings between odd-dimensional manifolds.

A result of E. G. Skljarenko [11] states that a map between compact ANR's which is locally acyclic almost everywhere (i.e., except on a set of dimension $\leq 0)$ is in fact locally acyclic except on a finite set. After slightly modifying Skljarenko's result in $\$ 4$, we prove in $\$ 5$ a finiteness theorem for maps $f$ between closed $K$-orientable manifolds ( $K$ a field): If $f$ is locally $(k-1)$-acyclic almost everywhere, and $2 k \geq n$, then $f$ is locally acyclic except on a finite set. A similar result holds with $2 k+1=n$ and a local Euler characteristic hypothesis. As applications some of the finiteness theorems of $\$ \$ 1,2$ are improved and a further generalization of Wright's theorem is given.

The paper concludes with $\S 6$, where we consider PL maps $f: X \rightarrow \rightarrow Y$ between finite polyhedra. A proof of the following elementary formula is sketched: Suppose $Y_{0}$ is a subpolyhedron of $Y$ such that $\chi\left(f^{-1}(y)\right)=c_{0}$ for all $y \in Y_{0}$ and $\chi\left(f^{-1}(y)\right)=c$ for all $y \in Y \backslash Y_{0}$; then $\chi(X)=c \chi(Y)+\left(c_{0}-c\right) \chi\left(Y_{0}\right)$.

1. Definitions and a review of the even-dimensional case. For the following definitions suppose that $f: M \rightarrow \rightarrow N$ is a map with compact point-inverses. Recall three "singular sets"

$$
\begin{aligned}
A_{i}(f ; R) & =\left\{y \in N \mid f^{-1}(y) \text { does not have property } i-u v(R)\right\}, \\
A^{i}(f ; R) & =\left\{y \in N \mid \widetilde{H}^{i}\left(f^{-1}(y) ; R\right) \neq 0\right\}, \\
C_{f} & =\left\{y \in N \mid f^{-1}(y) \text { is not cellular in } M\right\} .
\end{aligned}
$$

(A compact set $X \subset M$ has property $i-u v(R)$ if, for any open set $U$ containing $X$, there is an open set $V$ with $X \subset V \subset U$ such that $\widetilde{H}_{i}(V ; R) \rightarrow \widetilde{H}_{i}(U ; R)$ is zero. An equivalent condition is that the reduced Skljarenko homology of $X$ vanish in dimension $i$; see [12].) The map $f$ is called strongly acyclic in dimension $i$ (over $R$ ) if $A_{i}(f ; R)=\varnothing$ and strongly acyclic (over $R$ ) if $A(f ; R)=\varnothing$, where

$$
A(f ; R)=\bigcup_{i \geq 0} A_{i}(f ; R)=\bigcup_{i \geq 0} A^{i}(f ; R) .
$$


The first equality is a definition. The second follows from (3.3) of [7]. The above terminology is slightly different from that used in [9], where the following is proved:

Theorem 1.1. Suppose $f: M^{n} \rightarrow \rightarrow N^{n}$ is a proper map between $R$-orientable $n \cdot m a n i f o l d s$ and that $f$ is strongly acyclic in dimensions less than $k$.

(1) If $2 k>n$ then $f$ is strongly acyclic.

(2) If $2 k=n$ then $A(f ; R)$ is a locally finite subset of $N^{n}$.

Below we give analogues of (1) for $2 k=n$ and $2 k+1=n(\$ 3)$ and (2) for $2 k+1=n(\$ 2)$. Another even-dimensional result we shall analogize is the following, proved in [7].

Theorem 1.2. Suppose $f: M^{n} \rightarrow \rightarrow N^{n}$ is a proper map between n-manifolds and that $f^{-1}(y)$ bas property $U V^{k-1}$ for eacb $y \in N^{n}$.

(1) If $2 k>n \neq 4$ then $f$ is cellular (when $n=3$, we need to assume eacb $\mathrm{f}^{-1}(y)$ bas a neighborbood containing no fake cubes).

(2) If $2 k=n \neq 4$ then $C_{f}$ is a locally finite set in $N^{n}$.

See [1] or [7] for explanations of the terminology used in (1.2).

Conventions. A manifold is understood to be a connected, locally Euclidean metric space (without boundary points). $R$ always means a principal ideal do. main. A double arrow $M \rightarrow \rightarrow N$ indicates a surjective map. Otherwise, our notation is that of [14].

2. Finiteness theorems in odd dimensions. We will use local Euler characteristic assumptions in this section. When $G$ is a module over our PID $R$, we define rank $G$ to be the minimum number of generators of $\operatorname{Hom}_{R}(G, R)$, i.e., the rank of the free part of $G$. If and only if $X$ is a compactum with rank $\breve{H}^{i}(X ; R)$ finite for all $i$ and zero for all but finitely many $i$, we write

$$
\chi(X ; R)=\sum_{i}(-1)^{i} \text { rank } \check{H}^{i}(X ; R) .
$$

The question of dependence on $R$ will be ignored.

Theorem 2.1. Suppose that $f: M^{2 k+1} \rightarrow \rightarrow N^{2 k+1}$ is a proper map between $R$-orientable manifolds. If $f$ is strongly acyclic in dimensions less than $k$ and if $\chi\left(f^{-1}(y) ; R\right)=1$ for each $y \in N^{2 k+1}$, then $A(f ; R)$ is a locally finite set in $N^{2 k+1}$.

Proof. The set $A^{k+1}(f ; R)$ is locally finite by Theorem 2.3 of $[0]$ and 
$A^{q}(f ; R)=\varnothing$ for $q \neq k, k+1$ by $(1.3)$ of $[9]$. We claim that $A(f ; R)=A^{k+1}(f ; R)$. To see this, let $y \in N \backslash A^{k+1}(f ; R)$. Then

$$
\chi\left(f^{-1}(y) ; R\right)=1+(-1)^{k} \operatorname{rank} \breve{H}^{k}\left(f^{-1}(y) ; R\right)
$$

and hence rank $\check{H}^{k}\left(f^{-1}(y) ; R\right)=0$. Since $f^{-1}(y)$ has property $(k-1)-u v(R)$, it follows from the homology/cohomology universal coefficient theorem that $\breve{H}^{k}\left(f^{-1}(y) ; R\right)=0$. Therefore $y \in N \backslash A(f ; R)$.

Remarks. 1. The assumption $\chi\left(f^{-1}(y) ; R\right)=1$ in (2.1) can be weakened to the inequality $\chi\left(f^{-1}(y) ; R\right) \geq 1$ ( $k$ odd) or $\chi\left(f^{-1}(y) ; R\right) \leq 1$ (k even) without altering the conclusion. In the context of $(2.1)$, where $\chi\left(f^{-1}(y) ; R\right)=1+(-1)^{k} \beta_{k}$ $+(-1)^{k+1} \beta_{k+1}$, this means assuming $\beta_{k} \leq \beta_{k+1}$ instead of $\beta_{k}=\beta_{k+1}$. (Note that $\beta_{k+1}$ is finite; see the remark following the proof of (2.2) in [0].) Under this weaker assumption, one can use the conclusion of the theorem to show that $\chi\left(f^{-1}(y) ; R\right)=1$ for all $y$ so that the weaker hypothesis never arises in practice.

2. Under the hypothesis of (2.1), take $R=Z$ or $Z_{2}$. Then $\operatorname{deg} f= \pm 1$, so $f_{*_{i}}$ is an isomorphism for $i \neq k, k+1$ and an epimorphism for all $i$. Moreover,

$$
\operatorname{ker} f_{* k} \simeq \bigoplus_{y \in N} \check{H}^{k+1}\left(f^{-1}(y) ; R\right) \text {, and } \operatorname{ker} f_{* k+1} \simeq \bigoplus_{y \in N} \breve{H}^{k}\left(f^{-1}(y) ; R\right) \text {. }
$$

See the analysis in $\$ 7$ of [0].

3. When $R=\mathbf{Z}$ or $\mathrm{Z}_{2}$, the orientability hypotheses in (2.1) may be dropped. See $\$ 4$ of [9].

The orem 2.2. Suppose $f: M^{2 k+1} \rightarrow \rightarrow N^{2 k+1}$ is a proper map between manifolds and that $f^{-1}(y)$ has property $U V^{k-1}$ for each $y \in N^{2 k+1}$. If $\chi\left(f^{-1}(y) ; Z_{2}\right)$ $=1$ for all $y \in N^{2 k+1}$, then $C_{f}$ is a locally finite set in $N^{2 k+1}$.

Proof. Suppose first that $k \geq 2$. We have $A\left(f ; \mathbf{Z}_{2}\right)$ locally finite by (2.1) and $A^{k+1}(f ; Z)$ locally finite by Theorem 2.3 of $[9]$. Let $F=A\left(f ; Z_{2}\right) \cup A^{k+1}(f ; Z)$. We claim that $F=A(f ; Z)$. To prove this, let $y \in N \backslash F$. Then

$$
\tilde{H}^{i}\left(f^{-1}(y) ; Z\right)=0 \text { for } i \neq k \text { and } \tilde{H}^{i}\left(f^{-1}(y) ; Z_{2}\right)=0 \text { for all } i \text {. }
$$

It follows from the universal coefficient theorem for Čech cohomology that $\breve{H}^{k}\left(f^{-1}(y) ; \mathbf{Z}\right)=0$. This proves the claim and the local finiteness of $A(f ; \mathbf{Z})$. Now we are finished since $f^{-1}(y)$ has $U V^{\infty}$ for each $y \in N \backslash A(f ; Z)$. (See $\$ 4$ of [7].)

Now assume $k=1$. Then $A\left(f ; \mathbf{Z}_{2}\right)$ is locally finite, hence zero-dimensional, so a result of [17] applies.

Remarks. 1. If $f$ is as in (2.2) with $M$ and $N$ closed manifolds, then we can find a closed, $(k-1)$-connected manifold $K$ such that $M$ is homeomorphic to the connected sum $N \# K$. Conversely, if $M=N \# K$, where $K$ is $(k-1)$-con- 
nected, we can construct a map $f: M \rightarrow \rightarrow N$ which satisfies (2.2). See the discussion in $\$ 7$ of [7].

2. An interesting aspect of (2.2) is that its statement makes no dimensional restrictions on the manifolds and thus seems to explain the "Wright phenomenon" (cf. [17]) as a statement about manifolds in general. The dichotomy between $k=$ 1 and $k>1$ occurs in the proof for two reasons: first, of course, the Poincaré conjecture, and second, the fact that $U V^{k-1}$ implies $1-U V$ in the latter situations while $U V^{0}$ is merely the statement that $f$ is monotone.

3. Both (1.1) and (2.1) can be generalized in the case where $R$ is a field by requiring only that $\operatorname{dim} A_{i}(f ; R) \leq 0$ for $i<k$ (and that $\operatorname{dim}\left\{y \in N \mid \chi\left(f^{-1}(y)\right) \neq 1\right\}$ $\leq 0$ in (2.1)). See $\$ 5$ below.

4. Some kind of hypothesis on $f^{-1}(y)$ in dimension $k$ is necessary in both (2.1) and (2.2). See $\$ 6$ of [7].

3. Nonalternating mappings. Suppose that $f: M \rightarrow N$ is a mapping. We shall say that $f$ is nonalternating in dimension $k$ provided that, for each pair, $y, z \in N$ with $y \neq z$, there exists a neighborhood $V$ of $f^{-1}(y)$ in $M$ such that $\widetilde{H}_{k}(V) \rightarrow \widetilde{H}_{k}\left(M-f^{-1}(z)\right)$ is the zero homomorphism. (Throughout $\S 3, R$ is assumed to be a fixed principal ideal domain, and all homology/cohomology is taken with coefficients in $R$. Otherwise, our notation follows that of previous sections.) Notice that if $M$ and $N$ are locally compact ANR's and each $f^{-1}(y)$ is compact, then "nonalternating in dimension zero" agrees with the classical notion of nonalternating. See [16].

Similarly, we shall say $f$ is weakly acyclic in dimension $k$ if each $f^{-1}(y)$ has a neighborhood $V$ in $M$ such that $\widetilde{H}_{k}(V) \rightarrow \widetilde{H}_{k}(M)$ is zero.

The following result is a corollary to R. Soloway's version of the Vietoris mapping theorem for singular homology. (See [13, Theorem 5].)

Theorem S. Suppose that $M$ and $N$ are locally compact ANR's and that $f: M \rightarrow \rightarrow N$ is a proper map which is strongly acyclic in dimensions less than k. Then $f_{*_{i}}: H_{i}(M) \rightarrow H_{i}(N)$ is an isomorpbism for $i \leq k-1$ and an epimorphism for $i=k$. If, in addition, $f$ is weakly acyclic in dimension $k$, then $f_{*_{k}}$ is an isomorphism.

We will be applying Theorem $S$ to certain types of maps between manifolds.

Theorem 3.1. Suppose $f: M^{n} \rightarrow \rightarrow N^{n}$ is a proper map between R-orientable $n-m a n i f o l d s, k<n$. If $f$ is strongly acyclic in dimensions less than $k$ and weakly acyclic in dimension $k$ then $\breve{H}^{i}\left(f^{-1}(y)\right)=0$ for $i \geq n-k$ and all $y \in N^{n}$.

Proof. Let $y \in N$, and consider 


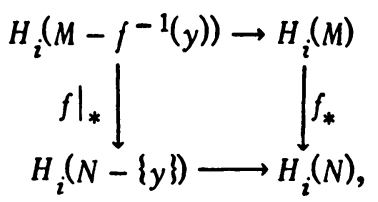

a commutative diagram. By Theorem $\mathrm{S}, f_{*}$ is an isomorphism when $i \leq k$ and $\left.f\right|_{*}$ is an isomorphism when $i<k$ and an epimorphism when $i=k$. Since the lower horizontal map is an isomorphism for $i<n$, we can conclude that the upper horizontal map is an isomorphism when $i<k$ and an epimorphism when $i=k$. It follows from the homology sequence of $\left(M, M-f^{-1}(y)\right)$ that $H_{i}\left(M, M-f^{-1}(y)\right)$ $=0$ for $i \leq k$; the result follows from duality.

Corollary 3.2. Suppose $f: M^{2 k} \rightarrow \rightarrow N^{2 k}$ is a proper map between R-orientable manifolds which is strongly acyclic in dimensions less than $k$. If $f$ is weakly acyclic in dimension $k$, then $f$ is strongly acyclic.

Corollary 3.2 is not surprising in view of the local finiteness of $A(f)$ noted above. We should point out, however, that one cannot conclude, in Theorem 3.1, that $f$ is strongly acyclic in dimension $k:$ there is a map $f: s^{2 k+1} \rightarrow \rightarrow S^{2 k+1}$ which is strongly acyclic in dimensions less than $k$ (and, obviously, weakly acyclic in dimension $k$ ) which is not strongly acyclic in dimension $k$. (See $\$ 6$ of [7].)

Changing from weakly acyclic to nonalternating, we can obtain an acyclicity criterion in odd dimensions as follows.

Theorem 3.3. Suppose $f: M^{2 k+1} \rightarrow \rightarrow N^{2 k+1}$ is a proper map between $R$ orientable manifolds. If $f$ is strongly acyclic in dimensions less than $k$ and nonalternating in dimension $k$, then $f$ is strongly acyclic.

Proof. Let $y \in N$. By (3.1), we have $\breve{H}^{i}\left(f^{-1}(y)\right)=0$ for $i>k$; and by $\S 3$ of [7] $\widetilde{H}^{i}\left(f^{-1}(y)\right)=0$ for $i<k$. It suffices, therefore, to show that $\check{H}^{k}\left(f^{-1}(y)\right)=0$. We claim first that

$$
H_{c}^{k}(M) \rightarrow \check{H}^{k}\left(f^{-1}(y)\right)
$$

is zero. For the proof, let $U$ be a neighborhood of $f^{-1}(y)$ such that

$$
\alpha: H_{k}(U) \rightarrow H_{k}(M)
$$

is zero, and let $V$ be a neighborhood of $f^{-1}(y)$ in $U$ such that

$$
\beta: H_{k-1}(V) \rightarrow H_{k-1}(U)
$$

is zero. Consider the commutative diagram below (from the universal coefficient theorem): 


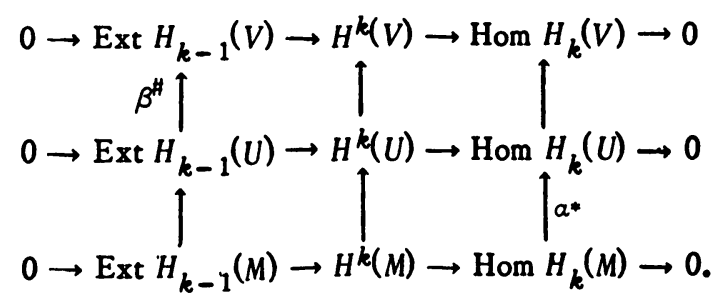

(The notation is as in $\$ 2$ of [7].) We have $\alpha^{*}=0$ and $\beta^{\#}=0$. Since the rows of the diagram are exact, it follows that $H^{k}(M) \rightarrow H^{k}(V)$ is zero, and hence that $H^{k}(M) \rightarrow \breve{H}^{k}\left(f^{-1}(y)\right)$ is zero. The claim now follows easily from the fact that $H^{k}(\hat{M}) \rightarrow H_{c}^{k}(M)$ is a functorial isomorphism $(k \neq 0)$, where $\hat{M}$ is the one-point compactification of $M$. (See $[14$, pp. 331, 332].) Now consider the diagram

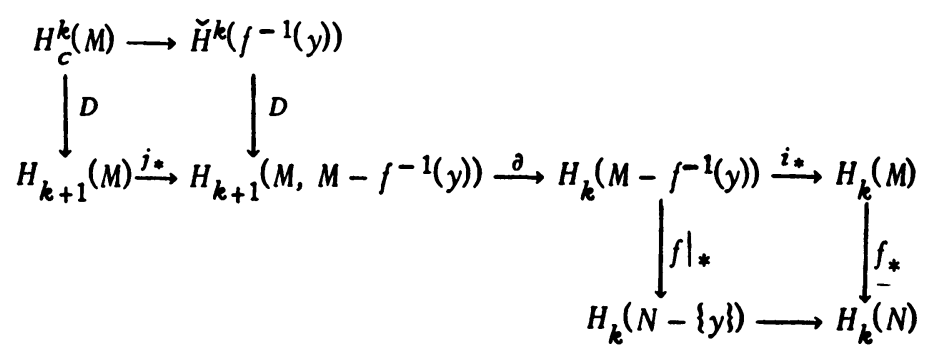

in which $D=$ duality isomorphism and both $f_{*}$ and $f_{*}$ are isomorphisms by Theorem $\mathrm{S}$. The diagram commutes up to sign, and the long row is exact. It follows that $i_{*}$ is an isomorphism and hence that $\partial=0$. The above paragraph implies that $j_{*}=0$, so we have

$$
0=H_{k+1}\left(M, M-f^{-1}(y)\right) \simeq \check{H}^{k}\left(f^{-1}(y)\right) .
$$

Therefore, $\widetilde{H}^{*}\left(f^{-1}(y)\right)=0$.

We conclude by remarking that there exist maps $f: s^{2 k} \rightarrow \rightarrow s^{2 k}$ which are strongly acyclic in dimensions $\leq k-2$, nonaltemating in dimension $k-1$, but not strongly acyclic in dimension $k-1$ : suspend the "join" example of $\$ 6$ of [7].

Remark. A technique of Soloway [13] can be used to conclude the properness of the map $f$, by merely assuming each point-inverse of $f$ is compact, in each of the situations (1.1), (1.2), (2.1), (2.2) and (3.2) (but definitely not in (3.1)).

Question. Suppose $f: M^{2 k+1} \rightarrow \rightarrow N^{2 k+1}$ is a map with compact point-inverses, strongly acyclic in dimensions less than $k$, and nonaltemating in dimension $k$. Is $f$ proper?

4. Almost acyclic mappings. The following theorem differs from a result of Skljarenko's [11] only in its dependence on the integer $k$. For the statement, we take $G$ to be a finitely generated $R$-module and $A^{q}(f ; G)$ to be the set of values $y$ for which $\check{H}^{q}\left(f^{-1}(y) ; G\right) \neq 0$. Following Skljarenko, if $A$ is a subset of the space 
$Y$, we define rd $A$ to be

$$
\text { rd } A=\max \{\operatorname{dim} C \mid C \text { is closed in } Y \text { and } C \subset A\}
$$

where $\operatorname{dim} C$ means the covering dimension of $C$.

Theorem 4.1. Let $f: X \rightarrow \rightarrow Y$ be a closed map between paracompact Hausdorff spaces. Suppose further that, for some integer $k \geq 0$, the following bold:

(i) $\check{H}^{q}(X ; G)$ is finitely generated for $q \leq k$;

(ii) $\check{H}^{q}(Y ; G)$ is finitely generated for $q \leq k+1$; and

(iii) $\operatorname{rd~} A^{q}(f ; G) \leq 0$ for $q \leq k$.

Then $A^{q}(f ; G)$ is finite for $q \leq \bar{k}$ and $\breve{H}^{q}\left(f^{-1}(y) ; G\right)$ is finitely generated for $q \leq$ $k$ and $y \in Y$.

The proof is based on that of Skljarenko and uses the Leray spectral sequence of $f$. We outline the major steps.

Lemma 4.2. Let $\left\{E_{r}^{* *}\right\}$ be a convergent first quadrant spectral sequence. If $E_{2}^{p, q}=0$ whenever $p>0$ and $0<q \leq k$, then there exists an exact sequence

$$
E_{2}^{1,0} \rightarrow \cdots \rightarrow E_{2}^{p, 0} \rightarrow H^{p} \rightarrow E_{2}^{0, p} \rightarrow E_{2}^{p+1,0} \rightarrow \cdots \rightarrow E_{2}^{k+2,0},
$$

the maps $E_{2}^{p, 0} \rightarrow H^{p} \rightarrow E_{2}^{0, p}$ being edge homomorphisms and $E_{2}^{0, p} \rightarrow E_{2}^{p+1,0}$ being the map $d_{2, p+1}^{0, p}$ of [6].

Proof. Simply apply three propositions from Chapter XV of [6]: (5.7), (5.9), and (5.9a).

Now suppose that $f: X \rightarrow Y$ is a closed, surjective map between paracompact Hausdorff spaces and assume that $\operatorname{rd} A^{q}(f ; G) \leq 0, q \leq k$. Following Skljarenko [11], we define

$$
\mathcal{G}^{q}=R^{q} / G, \quad 1 \leq q \leq k, \quad \text { and } \quad \varrho^{0}=R^{0} f G / G .
$$

(Here, $R^{q} f$ is the $q$ th right derived functor of the direct image functor. See [5].) Finally, let $\Gamma^{q}=\Gamma\left(Y, \varrho^{q}\right), 0 \leq q \leq k$, i.e., $\Gamma^{q}$ is the module of sections of the sheaf $\oint^{q}$ over $Y$.

Lemma 4.3. Under the above assumptions, there exists an exact sequence

$$
\begin{aligned}
0 \rightarrow \check{H}^{0}(Y ; G) & \rightarrow \cdots \rightarrow \check{H}^{q}(Y ; G) \rightarrow \check{H}^{q}(X ; G) \rightarrow \Gamma^{q} \rightarrow \check{H}^{q+1}(Y ; G) \\
& \rightarrow \cdots \rightarrow \check{H}^{k+2}(X ; G) .
\end{aligned}
$$

Proof. The proof is the same as Skljarenko's. His arguments show that $\left\{E_{r}^{* *}\right\}$ satisfies the hypothesis of (4.2), where $\left\{E_{r}^{* *}\right\}$ is the Leray spectral sequence of $f$. This fact yields most of the required sequence, since $E_{2}^{P, 0}=$ $\breve{H}^{P}(Y ; G), H^{P}=\check{H}^{P}(X ; G)$, and $E_{2}^{0, P}=\breve{H}^{0}\left(Y ; R^{P} f G\right)=\Gamma^{P}$. The first few terms 
are constructed in the present situation just as they are in [11].

Remark. For $q \leq k$ the module $\Gamma^{q}$ is finitely generated if and only if $A^{q}(f ; G)$ is finite and each $\breve{H}^{q}\left(f^{-1}(y) ; G\right)$ is finitely generated, $y \in Y$. (See [11].) Hence (4.1) follows from (4.3).

The introduction of "relative dimension" is an empty generalization in the case of mappings between manifolds, as we now show that $\mathrm{rd}$ and dim agree on $A^{i}(f ; G)$. (This was suggested by D. R. McMillan, Jr., who pointed out that the same is true for $A_{i}(f ; G)$.)

Theorem 4.4. Suppose $f: X \rightarrow \rightarrow Y$ is a proper map between metric spaces, with $X$ a locally compact $A N R$. Then $A^{i}(f ; G)$ is a countable union of closed subsets of $Y$, and bence $\operatorname{rd} A^{i}(f ; G)=\operatorname{dim} A^{i}(f ; G)$.

Proof. If $\mathcal{U}$ is an open cover of $Y$, define $B(\mathcal{U})$ to be the set $\{x \in X \mid$ if $f(x) \in U \in \mathcal{U}$ then $\widetilde{H}^{i}\left(f^{-1}(U) ; G\right) \rightarrow \widetilde{H}^{i}\left(f^{-1} f(x) ; G\right)$ is not zero\}. We claim that $B(\mathcal{U})$ is a closed subset of $X$. To see this, suppose $x$ is a limit point $B(\mathcal{U})$, and suppose $f(x) \in U \in \mathcal{U}$. Let $V_{1}, V_{2}, \cdots$ be open sets in $Y$ such that $\bar{V}_{n+1} \subset$ $V_{n} \subset U$ for all $n$ and $f(x)=\bigcap_{n} \bar{V}_{n}$. Choose points $y_{n} \in V_{n} \cap f(B(\mathcal{U}))$ for each $n$. Considering the diagram

$$
\widetilde{H}^{i}\left(f^{-1}\left(V_{n}\right)\right) \leftarrow \tilde{H}^{i}\left(f^{-1}(U)\right)
$$

one sees easily that $\tilde{H}^{i}\left(f^{-1}(U)\right) \rightarrow \tilde{H}^{i}\left(\bar{f}^{-1}\left(V_{n}\right)\right)$ is not zero for each $n$, since $f^{-1}\left(y_{n}\right) \subset B(\mathcal{U})$. If we choose $\left\{V_{n}\right\}$ with the additional property that image $\left[\tilde{H}^{i}\left(f^{-1}\left(V_{n}\right)\right) \rightarrow \tilde{H}^{i}\left(f^{-1}\left(V_{n+1}\right)\right)\right]$ is finitely generated for each $n$, then it follows that the map

$$
\widetilde{H}^{i}\left(f^{-1}(U)\right) \rightarrow \lim _{n} \widetilde{H}^{i}\left(f^{-1}\left(V_{n}\right)\right) \simeq \widetilde{H}^{i}\left(f^{-1} f^{(}(x)\right)
$$

1s nonzero. That $\left\{V_{n}\right\}$ may be so chosen follows from an argument similar to the one for (2.1) of [9]. Therefore $x \in B(U)$.

Taking an appropriate sequence of open covers of $Y$ shows that $f^{-1}\left(A^{i}(f ; G)\right)$ (and, hence, $A^{i}(f ; G)$ ) is a countable union of closed sets.

The second part of the conclusion follows from the "Sum Theorem" for dimension.

5. Almost acyclic mappings between manifolds. In this section we let $K$ be a field. We conjecture that $K$ could be replaced by $R$ in (5.1).

Theorem 5.1. Let $f: M^{m} \rightarrow \rightarrow N^{n}$ be a map between closed, K-orientable 
manifolds, $k<n$. Suppose $\operatorname{dim} A^{q}(f ; K) \leq 0$ for $q<k$. Then $A^{q}(f ; K)$ is finite whenever $q<k$ or $q \geq m-k$. Therefore, if $2 k \geq m, A(f ; K)$ is finite.

Proof. By (4.1), $A^{q}(f)$ is finite for $q<k_{0}$ (We suppress $K$ from notation in the proof.) Let

$$
V=N-\bigcup_{q<k} A^{q}(f), \quad U=f^{-1}(V)
$$

Applying Theorem 1.3 of [9], we see that $A^{q}(f \mid U)=\varnothing$ for $q<k$ and $q>m-k$ (and, hence, $A^{q}(f)$ is finite in these ranges). Also, Theorem 2.3 of [9] implies that $A^{m-k}(f \mid U)$ is a locally finite subset of $U$.

We wish to show that $A^{m-k}(f \mid U)$ is actually finite. Suppose $Y$ is any finite subset of $A^{m-k}(f \mid U)$, and let $X=f^{-1}(Y)$. By Theorem 1.1 of [9], the inclusioninduced map $H_{c}^{m-k}(U) \rightarrow \breve{H}^{m-k}(X)$ is epic. Let $\hat{U}$ be the one-point compactification of $U$. We have the following diagram, each map being induced by inclusion.

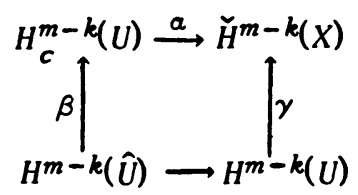

The map $\alpha$ is epic, as noted above, and $\beta$ is an isomorphism (assuming $m-k \neq$ $0)$. Therefore, $\alpha \beta$ is epic, and hence $\gamma$ is epic. Let $d$ be the dimension of $H^{m-k}(U)$. (We show in the next paragraph that $d$ is finite.) Since

$$
\check{H}^{m-k}(X)=\bigoplus_{y \in Y} \check{H}^{m-k}\left(f^{-1}(y)\right),
$$

the cardinality of $Y$ is no greater than $d$; therefore, $A^{m-k}(f \mid U)$ is finite. It follows that

$$
A^{m-k}(f) \subset\left[A^{m-k}(f \mid U) \cup \underset{q<k}{\bigcup} A^{q(f)}\right]
$$

a finite set.

It remains to show that $H^{m-k}(U)$ is finitely generated. For this, it suffices to show that $H_{m-k}(U)$ is finitely generated. A portion of the homology sequence of $(M, U)$ looks like

$$
H_{m-k+1}(M, U) \rightarrow H_{m-k}(U) \rightarrow H_{m-k}(M)
$$

where

$$
H_{m-k+1}(M, U) \simeq \check{H}^{k-1}\left(f^{-1}\left(\bigcup_{q<k} A^{q}(f)\right)\right)=\underset{y \in A^{k-1}(f)}{\bigoplus}\left\{\breve{H}^{k-1}\left(f^{-1}(y)\right)\right\} .
$$


Since $A^{k-1}(f)$ is finite and $\breve{H}^{k-1}\left(f^{-1}(y)\right)$ is finitely generated (by (4.1)) for each $y \in N$, the result follows.

The following is an odd-dimensional analogue of the last statement in (5.1).

Theorem 5.2. Suppose that $f: M^{2 k+1} \rightarrow \rightarrow N^{2 k+1}$ is a map between closed, $K$-orientable manifolds. Suppose further that $\operatorname{dim} A^{q}(f ; K) \leq 0$ for $q<k$ and that $\operatorname{dim}\left\{\left.y\right|_{\chi}\left(f^{-1}(y) ; K\right) \neq 1\right\} \leq 0$. Then $A(f ; K)$ is finite.

Proof. By (5.1) the sets $A^{q}(f)$ are finite whenever $q \neq k$. We have the inclusion

$$
A^{k}(f) \subset\left[\left\{y \mid \chi\left(f^{-1}(y)\right) \neq 1\right\} \cup \bigcup_{q \neq k} A^{q(f)}\right],
$$

so $\operatorname{dim} A^{k}(f) \leq 0$. The result follows from (4.1).

Applying Wright's results (Theorem 1 of [17]) again we obtain what may be the ultimate generalization of his theorem, at least for maps between closed 3manifolds.

Corollary 5.3. Let $f: M^{3} \rightarrow \rightarrow N^{3}$ be a map between closed 3-manifolds. If there exists a zero-dimensional set $Z \subset N^{3}$ sucb that $f^{-1}(y)$ is connected and $\chi\left(f^{-1}(y) ; Z_{2}\right) \geq 1$ for each $y \in N^{3} \backslash Z$, then $C_{f}$ is a finite set. Consequently $M^{3}$ is the connected sum of $N^{3}$ and some closed 3-manifold.

The proof of (5.3) uses Remark 1 of $\S 2$ as applied to the proof of (5.2). Note, incidentally, that there are monotone maps $b: s^{3} \rightarrow \rightarrow S^{3}$ with $\chi\left(b^{-1}(y)\right) \geq-1$ for each $y$ and $C_{b}$ an arc in $S^{3}$.

Question. If $f: M^{3} \rightarrow \rightarrow N^{3}$ is a monotone map with $\chi\left(f^{-1}(y)\right) \geq 0$ for each $y$, must $C_{f}$ be finite?

6. PL mappings and the Euler characteristic. In the preceding sections local Euler characteristic assumptions were used several times, and a natural question seems to appear: If $f: X \rightarrow \rightarrow Y$ is a map between compact ANR's and $\chi\left(f^{-1}(y)\right)=c$ for all $y \in Y$, where $c$ is constant, what can be said about $\chi(X)$ as related to $\chi(Y)$ ? There is an easy answer when everything is PL, and we sketch this answer here.

Theorem 6.1. Suppose that $f: X \rightarrow \rightarrow Y$ is a $P L$ map between (finite) polybedra and that $Y_{0}$ is a subpolybedron of $Y$. If there are integers $c_{0} c_{0}$ sucb that $\chi\left(f^{-1}(y)\right)=c$ for $y \in Y \backslash Y_{0}$ and $\chi\left(f^{-1}(y)\right)=c_{0}$ for $y \in Y_{0}$, then

$$
\chi(X)=c \chi(Y)+\left(c_{0}-c\right) \chi\left(Y_{0}\right)
$$

Here, $\chi(X)$ denotes the usual Euler characteristic $\chi(X$; rational numbers). 
The proof of (6.1) requires the following calculation, in which \#S denotes the cardinality of the set $S$ and $\hat{\Delta}$ denotes the barycenter of $\Delta$.

Lemma 6.2. If $f: K \rightarrow \rightarrow \Delta^{n}$ is a simplicial map of the finite complex $K$ onto an $n$-simplex then

$$
\sum_{j}(-1)^{j} \#\left\{\sigma^{j} \in K \mid f\left(\sigma^{j}\right)=\Delta^{n}\right\}=(-1)^{n} \chi\left(f^{-1}\left(\hat{\Delta}^{n}\right)\right) .
$$

Proof. Let $H^{j}=\left\{\sigma^{n+j} \in K \mid f\left(\sigma^{n+j}\right)=\Delta^{n}\right\}$ and $H=\bigcup_{j} H_{j}$. Now $f^{-1}\left(\hat{\Delta}^{n}\right)$ has a natural triangulation as a subcomplex of a first derived subdivision of $K$, but we want instead a cell structure determined by $K$ as follows. Associate with each $\sigma \in H^{j}$ the set $\Gamma(\sigma)=f^{-1}\left(\hat{\Delta}^{n}\right) \cap \sigma$. Notice that $\Gamma\left(\sigma^{n+j}\right)$ is a $j$-dimensional cell and in fact $\Gamma=\{\Gamma(\sigma) \mid \sigma \in H\}$ is a cell complex whose underlying space is $f^{-1}\left(\hat{\Delta}^{n}\right)$. The Euler characteristic of $f^{-1}\left(\hat{\Delta}^{n}\right)$ can be computed using this cell structure, and we obtain

$$
\chi\left(f^{-1}\left(\hat{\Delta}^{n}\right)\right)=\sum_{j}(-1)^{j} \#\left\{\gamma^{j} \in \Gamma\right\}=\sum_{j}(-1)^{j} \# H^{j}=(-1)^{n} \sum_{j}(-1)^{n+j} \# H^{j},
$$

which completes the proof.

Proof of (6.1). First assume that $\left(K, K_{0}\right)$ and $\left(L, L_{0}\right)$ are triangulations of $\left(X, f^{-1}\left(Y_{0}\right)\right)$ and $\left(Y, Y_{0}\right)$, respectively, such that $f$ and $f \mid f^{-1}\left(Y_{0}\right)$ are simplicial.

Assume as a special case that $Y_{0}=\varnothing$. Let $\sigma^{n}$ be a top-dimensional simplex of $L, L_{1}=L \backslash\left\{\sigma^{n}\right\}$, and $K_{1}=f^{-1}\left(L_{1}\right)$. We have $\chi(L)=\chi\left(L_{1}\right)+(-1)^{n}$ and, by $(6.2) \chi(K)=\chi\left(K_{1}\right)+(-1)^{n} c$. By induction, we may assume that $\chi\left(K_{1}\right)=$ $c \chi\left(L_{1}\right)$, so $\chi(K)=c \chi\left(L_{1}\right)+(-1)^{n} c=c \chi(L)$.

Now we prove the theorem assuming $Y_{0} \neq \varnothing$ by induction on the number of simplexes of $L \backslash L_{0}$. The case $L \backslash L_{0}=\varnothing$ follows from the special case above, so we proceed to the inductive step. Let $r^{n}$ be a top-dimensional simplex of $L \backslash L_{0}, L_{1}=L \backslash\left\{r^{n}\right\}$, and $K_{1}=f^{-1}\left(L_{1}\right)$. Using (6.2) and the inductive hypothesis we have

$$
\begin{aligned}
\chi(K) & =\chi\left(K_{1}\right)+(-1)^{n} c=c \chi\left(L_{1}\right)+\left(c_{0}-c\right) \chi\left(L_{0}\right)+(-1)^{n} c \\
& =c\left(\chi(L)-(-1)^{n}\right)+\left(c_{0}-c\right) \chi\left(L_{0}\right)+(-1)^{n} c \\
& =c \chi(L)+\left(c_{0}-c\right) \chi\left(L_{0}\right) .
\end{aligned}
$$

Remarks. Other similar results follow from the same kind of argument. For example, one can replace $\chi^{(-;}$rationals $)$by $[\chi(-; \text { rationals })]_{q}$ throughout, where [ ] $q$ denotes equivalence class modulo $q$ and the formula is interpreted in $\mathbf{Z}_{q}$. As another example, one can show the following: If $f: M^{n} \rightarrow \rightarrow N^{n}$ is a PL map between closed, orientable PL manifolds such that $\chi\left(f^{-1}(y)\right)=c$ for all $y \in N^{n}$, 
then $\operatorname{deg} f= \pm c$. For a more sophisticated mod 2 version of this last statement, see [15].

Added in proof (March 10, 1974). The question at the end of $\$ 5$ has been answered affirmatively by T. Knoblauch.

\section{REFERENCES}

1. S. Armentrout, UV-properties of compact sets, Trans. Amer. Math. Soc. 143 (1969), 487-498. MR 42 \#8451.

2. Cellular decompositions of 3-manifolds that yield 3-manifolds, Mem. Amer. Math. Soc. No. 107 (1971).

3. E. G. Begle, The Vietoris mapping theorem for bicompact spaces, Ann. of Math. (2) 51 (1950), 534-543. MR 11, 677.

4. R. H. Bing, The monotone mapping problem, Topology of Manifolds (Proc. Inst., Univ. of Georgia, Athens, Ga., 1969), Markham, Chicago, Ill., 1970, pp. 99-115. MR 43 \#1136.

5. G. E. Bredon, Sheaf theory, McGraw-Hill, New York, 1967. MR 36 \#4552.

6. H. Cartan and S. Eilenberg, Homological algebra, Princeton Univ. Press, Princeton, N. J., 1956. MR 17, 1040.

7. R. C. Lacher, Cellularity criteria for maps, Michigan Math. J. 17 (1970), 385396. MR 43 \#5539.

8. - Finiteness theorems in the study of mappings between manifolds, Proc. Conf. Top. (Univ. of Oklahoma, 1972), Dept. of Math., University of Oklahoma, Norman, 1972, pp. 79-96.

9. R. C. Lacher and D. R. McMillan, Jr., Partially acyclic mappings between manifolds, Amer. J. Math. 94 (1972), 246-266. MR 46 \#898.

10. L. C. Siebenmann, Approximating cellular maps by homeomorphisms, Topology 11 (1972), 271-294. MR $45 \# 4431$.

11. E. G. Skljarenko, Almost acyclic mappings, Mat. Sb. 75 (117) (1968), 296-302 = Math. USSR Sb. 4 (1968), 267-272. MR 37 \#4806.

12. - Homology theory and the exactness axiom, Uspehi Mat. Nauk 24 (1969), no. 5 (149), 87-140 = Russian Math. Surveys 24 (1969), no. 5, 91-142. MR 41 \#7676.

13. R. Soloway, Somewhere acyclic mappings of manifolds are compact, $\mathrm{Ph} . \mathrm{D}$. Thesis, University of Wisconsin, 1971.

14. E. H. Spanier, Algebraic topology, McGraw-Hill, New York, 1966. MR 35 \#1007.

15. D. Sullivan, Combinatorial invariants of analytic spaces, Proc. of Liverpool Singularities-Sympos., I (1969/70), Lecture Notes in Math., vol. 192, Springer, Berlin, 1971, pp. 165-168. MR 43 \#4063.

16. G. T. Whyburn, Analytic topology, Amer. Math. Soc. Colloq. Publ., vol. 28, Amer. Math. Soc., Providence, R. I., 1942. MR 4, 86.

17. A. H. Wright, Mappings from 3-manifolds onto 3-manifolds, Trans. Amer. Math. Soc. 167 (1972), 479-495.

DEPARTMENT OF MATHEMATICS, FLORIDA STATE UNIVERSITY, TALLAHASSEE, FLORIDA 323.06 (Current address)

DEPARTMENT OF MATHEMATICS, UNIVERSITY OF WARWICK, COVENTRY, ENGLAND 